\title{
Performance Aware Bit Plane Allocation
}

\author{
Gwanggil Jeon \\ Department of Embedded Systems Engineering, Incheon National University \\ 119 Academy-ro, Yeonsu-gu, Incheon 406-772, Korea \\ gjeon@inu.ac.kr
}

\begin{abstract}
This paper discusses bit plane allocation performance. The bit plane images are generated by bit plane slicing. The bit plane slicing is the process of conversion from original image to multilevel image. Normally, the number of multilevel is two. By bit plane slicing, a given image is sliced into n-level planes, where importance of planes is different. For example, the $0^{\text {th }}$ bit plane is the least significant one and the $7^{\text {th }}$ bit plane is the most significant one. Five metrics were used for evaluating bit plane, CMSE, CPSNR, $S$-CIELAB, FSIM and file size. Simulation results show the performance in each metrics.
\end{abstract}

Keywords: image processing, bit plane allocation, digital color image, image size, performance metric, image quality

\section{Introduction}

The bit plane slicing method uses a digital discrete image which is a set of bits point to a provided bit plane in each of the binary numbers depicting the signal [1-3]. The principal goal of bit plane slicing is to highlight contribution of given image, rather than highlight the gray level image [4-8]. Gray level images are composed of eight binary planes, which are ranging from $0^{\text {th }}$ to $7^{\text {th }}$ planes.

Separating a digital discrete image into its bit plane is efficient way to analyze importance of bit plane $[9,10]$. The $0^{\text {th }}$ bit plane is assumed to be the last bit of the image. This bit plane does not provide important information of the image, and therefore it is called as the least significant bit (LSB). On the other hand, the most important bit plane is $7^{\text {th }}$ bit, which is called as the most significant bit (MSB). There are few applications for bit plane. For instance, in pulse code modulation sound encoding, the first bit in the sample indicates the sign of the function [11-20]. This implies that only 1 bit can determined the sign of the whole signal, and the half of amplitude is depending on particular bit (MSB). However, the last bit of the sample does not much affect the information, and may be considered as 'don't care' bit. Therefore, it is important to sustain MSB, and pay less attention on LSB. Table 1 shows an example of pulse code modulation [21-23].

In this paper, we analysis the performance of bit plane slicing where CMSE, CPSNR, S-CIELAB, FSIM and file size are provided. The paper is arranged as follows. In Section 2 , bit plane slicing and performance metrics are provided. Simulation results are given in Section 3. Finally, in Section 4, we present our conclusions. 
Table 1. Example of Pulse Code Modulation

\begin{tabular}{|c|c|c|}
\hline Bit allocation & Value & Importance of bit \\
\hline 111 & 7 & \multirow{8}{*}{$\begin{array}{l}\text { MSE: } 4 \text { (in intensity) } \\
\text { LSE: } 1 \text { (in intensity) } \\
\text { Middle bit: } 2 \text { (in } \\
\text { intensity) }\end{array}$} \\
\hline 110 & 6 & \\
\hline 101 & 5 & \\
\hline 100 & 4 & \\
\hline 011 & 3 & \\
\hline 010 & 2 & \\
\hline 001 & 1 & \\
\hline 000 & 0 & \\
\hline
\end{tabular}

\section{Proposed Method}

The bit plane slicing method slices given $n$-bit image into $n$ binary planes. The least and the most significant bits are bit plane 0 and bit plane 7, which are depicted as Figure 1.

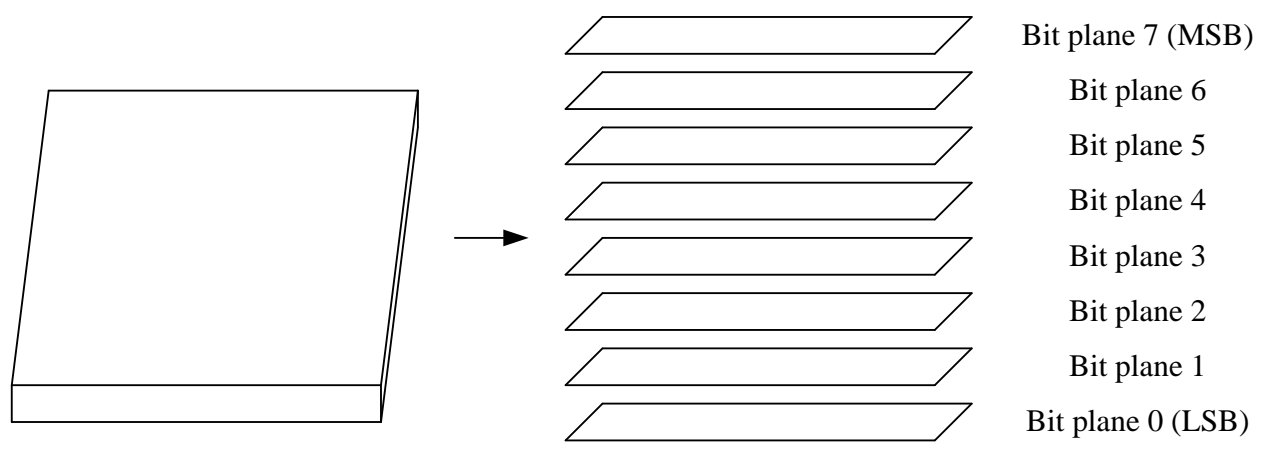

Figure 1. Bit Plane Slicing Representation of an Image ( $N=8)$

It is obvious that the intensity value of each pixel is described by an $n$-bit binary plane vector $(b p v): b p v_{7}, b p v_{6}, b p v_{5}, b p v_{4}, b p v_{3}, b p v_{2}$, and $b p v_{1}$.It is noted that channel resolution $n$ in Figure 1 is 8 . As $b v$ is represented as binary value, the intensity of $b p v$ is either 0 or 1 . And these values are accumulated in $n$ bit planes. Each bit plane can be considered as 2level images that are described by a binary matrix.

The $b p v$ value is represented as Eq. (1).

$$
b p v_{n}(i, j)=\text { remaining }\left(\frac{\text { floor }\left\{\frac{\operatorname{image}(i, j)}{2^{n}}\right\}}{2}\right) \text {, }
$$

Here, image $(i, j)$ is original image at $(i, j)$ pixel location. Parameter ' $b p v_{n}$ ' is bit plane information, and 'remaining' means remainder operator. Finally, 'floor $(k)$ ' is round the elements to $k$ nearest integers less than or equal to $k$.

The file size quantifies the size of the output file. Generally, it is described in the form of bytes. The real amount of disk space assesses by the file conditional upon the file 
system. Table 2 shows file size analysis, where Number of bytes allocated to the file is described. For instance, file size of bit plane $A$ to bit plane $B\left(B P_{A B}\right)$ has following file size.

Figures 2 and 3 show the accumulated bit plane example of bit plane slicing on 8-bit color image and Figure 3 shows separate plane example of bit plane slicing on 8-bit color image, respectively.

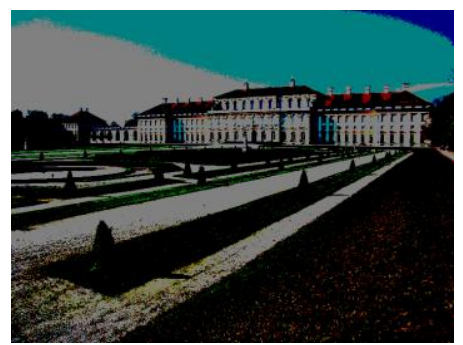

(a)

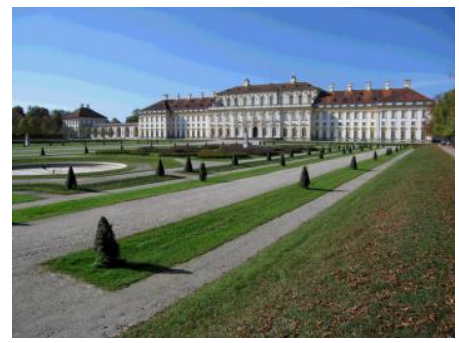

(d)

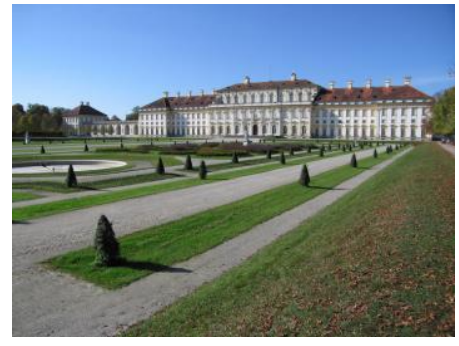

(g)

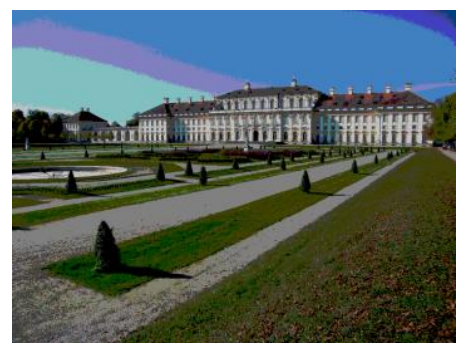

(b)

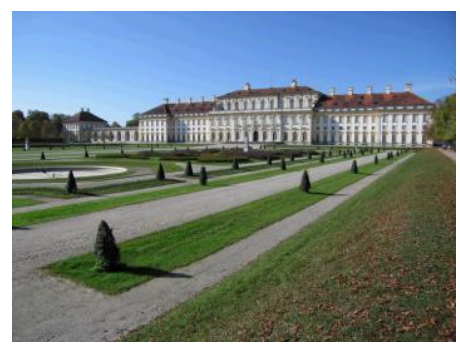

(e)

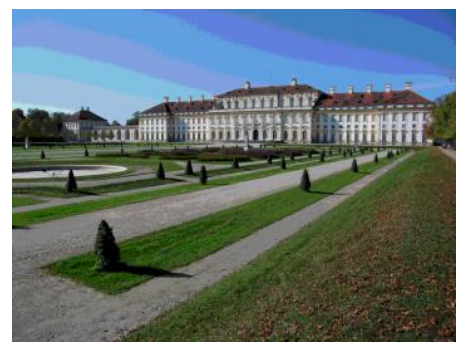

(c)

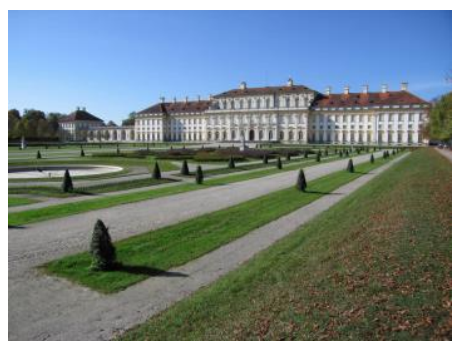

(f)

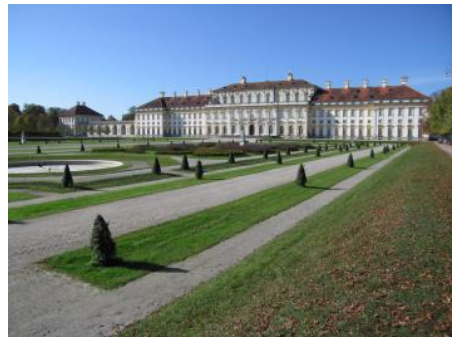

(h)

Figure 2. Accumulated Bit Plane Example of Bit Plane Slicing on 8-Bit Color Image: 31st LC Image: 31st LC Image: (A) Bit Plane 1 (LSB), (B) Bit Plane 1, (C) Bit Plane 2, (D) Bit Plane 3, (E) Bit Plane 4, (F) Bit Plane 5, (G) Bit Plane 6, And (H) Bit Plane 7 (MSB)

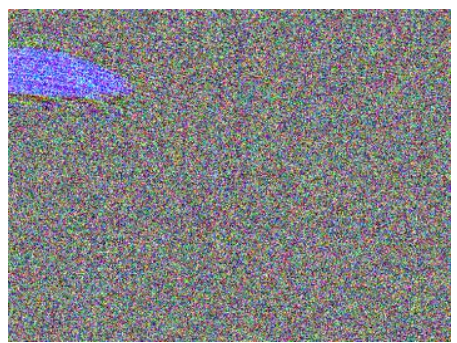

(a)

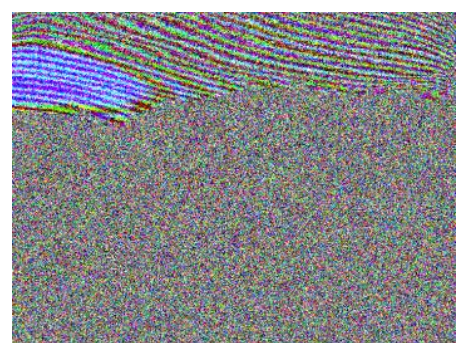

(b)

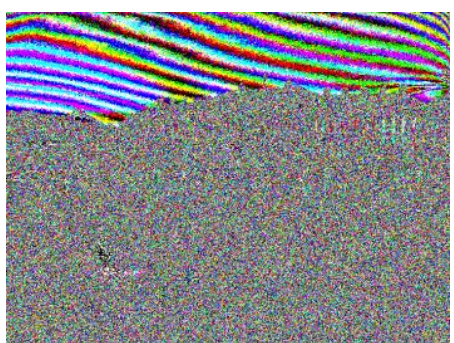

(c) 


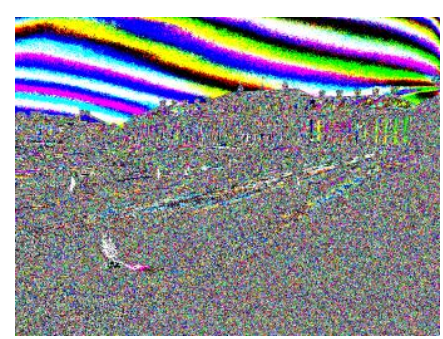

(d)

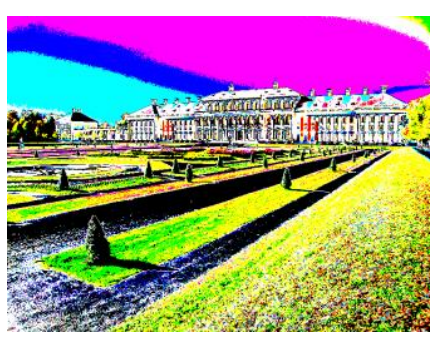

(g)

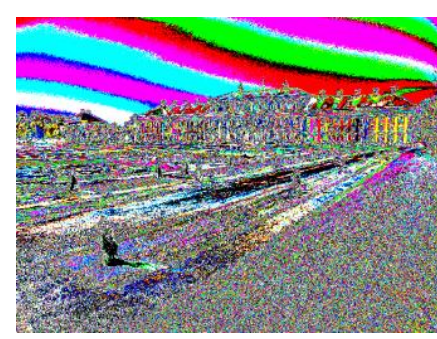

(e)

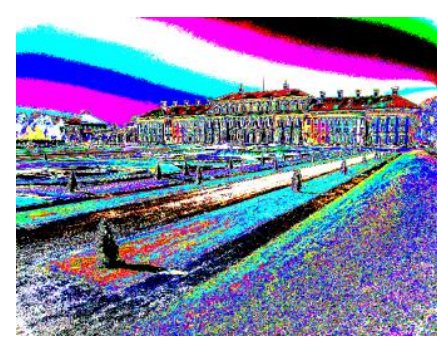

(f)

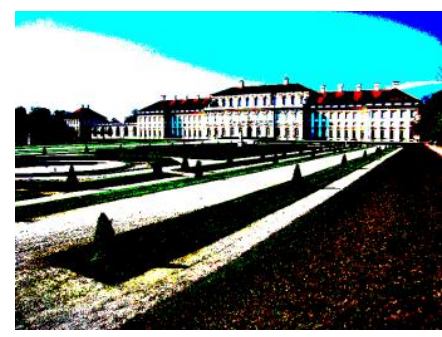

(h)

Figure 3. Separate Plane Example of Bit Plane Slicing on 8-Bit Color Image: 31st LC Image: (A) Bit Plane 1 (LSB), (B) Bit Plane 1, (C) Bit Plane 2, (D) Bit Plane 3, (E) Bit Plane 4, (F) Bit Plane 5, (G) Bit Plane 6, And (H) Bit Plane 7 (MSB)

Figure 4. Shows MSB Bit Plane in Three Channels: Red, Green, and Blue.

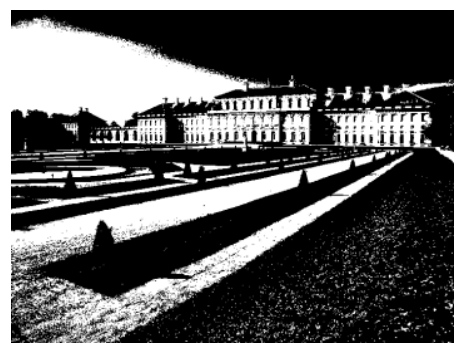

(a)

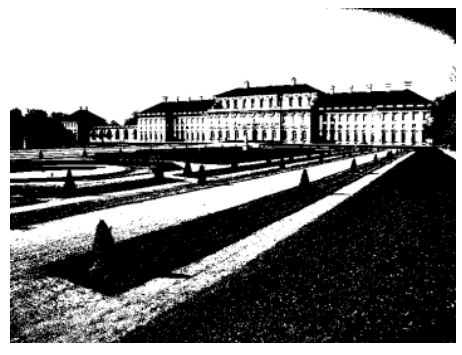

(b)

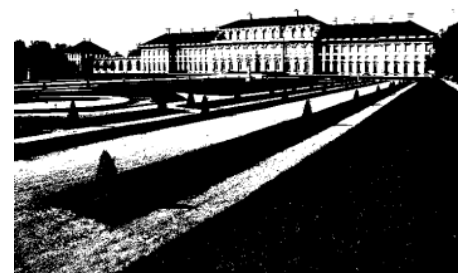

(c)

Figure 4. Red, Green, and Blue Channels of MSB: (A) Red, (B) Green, and (C) Blue 
Table 2. File Size Of \#31 LC Image

\begin{tabular}{|c|c|}
\hline$B P_{A B}$ & File size \\
\hline$B P_{77}$ & 49927 \\
\hline$B P_{67}$ & $122652\left(=4.0496\right.$ times of $\left.B P_{77}\right)$ \\
\hline$B P_{57}$ & $202183\left(=2.4566\right.$ times of $\left.B P_{77}\right)$ \\
\hline$B P_{47}$ & $293043\left(=5.8694\right.$ times of $\left.B P_{77}\right)$ \\
\hline$B P_{37}$ & $393672\left(=7.8850\right.$ times of $\left.B P_{77}\right)$ \\
\hline$B P_{27}$ & $512669\left(=10.2684\right.$ times of $\left.B P_{77}\right)$ \\
\hline$B P_{17}$ & $642987\left(=12.8785\right.$ times of $\left.B P_{77}\right)$ \\
\hline$B P_{07}$ & $719613\left(=14.4133\right.$ times of $\left.B P_{77}\right)$ \\
\hline
\end{tabular}

Traditional metric was assessed by gradient magnitude. The gradient operators can be expressed convolution masks, and their filters are shown in Table 3.

Table 3. Gradient Magnitude Filters

\begin{tabular}{|c|c|c|}
\hline \multirow{3}{*}{ Sobel } & $\frac{1}{4}\left[\begin{array}{ccc}1 & 0 & -1 \\
2 & 0 & -2 \\
1 & 0 & -1\end{array}\right] * f(k)$ & $\frac{1}{4}\left[\begin{array}{ccc}1 & 1 & 1 \\
0 & 0 & 0 \\
-1 & -1 & -1\end{array}\right] * f(k)$ \\
\hline \multirow{2}{*}{ Prewitt } & $\frac{1}{3}\left[\begin{array}{ccc}1 & 0 & -1 \\
1 & 0 & -1 \\
1 & 0 & -1\end{array}\right] * f(k)$ & $\frac{1}{3}\left[\begin{array}{ccc}1 & 1 & 1 \\
0 & 0 & 0 \\
-1 & -1 & -1\end{array}\right] * f(k)$ \\
\hline \multirow{3}{*}{ Scharr } & $\frac{1}{16}\left[\begin{array}{ccc}3 & 0 & -3 \\
10 & 0 & -10 \\
3 & 0 & -3\end{array}\right] * f(k)$ & $\frac{1}{16}\left[\begin{array}{ccc}3 & 10 & 3 \\
0 & 0 & 0 \\
-3 & -10 & -3\end{array}\right] * f(k)$ \\
\hline
\end{tabular}

In [24], authors proposed perceptual color difference metric, which is S-CIELAB, also known as perceptual color fidelity metric. In general, color difference is esteemed by a lot of factors: cone sensitivity of the eyes, ambient illumination, and spatial pattern of the targets. The S-CIELAB computation has two components, color transformation and the spatial filtering steps. Then, results are sent to CIELAB Delta E calculation.

$$
\Delta E_{a b}^{*}=\sqrt{\left(\Delta L^{*}\right)^{2}+\left(\Delta a^{*}\right)^{2}+\left(\Delta b^{*}\right)^{2}} .
$$

Another metric is Feature SIMilarity Index (FSIM) [25-27]. In general, image quality assessment metrics pursue to utilize computational models to assess the image quality correctly with visual evaluations. There have been few well known metrics such as structural-similarity (SSIM), which is pixel-based method and assess structure. However, SSIM considers black and white image, and rather is not suitable for multi-channel (color) image. The FSIM is based on human visual system (HVS). Output of FSIM ranges from 0 (poorest) to 1 (best). In this work, we use FSIM to assess the performance of each bit plane. We used LC imageset. The equation of $\mathrm{FSIM}_{\mathrm{C}}$ is determined as follows, 


$$
\begin{aligned}
\text { FSIM }_{C} & =\frac{\sum_{x \in \Omega} S_{P C}(x) S_{G}(x)\left\{S_{I}(x) S_{Q}(x)\right\}^{\lambda} P C_{m}(x)}{\sum_{x \in \Omega} P C_{m}(x)} \\
& =\frac{\sum_{x \in \Omega} S_{L}(x)\left\{S_{L}(x)\right\}^{\lambda} P C_{m}(x)}{\sum_{x \in \Omega} P C_{m}(x)} .
\end{aligned}
$$

where $\lambda>0$ is the parameter used to adjust the importance of the chromatic components.

\section{Simulation Results}

In this section, we used 20 LC images, which have $540 \times 720$ resolution. Used images are \#41 to \#60 LC images. There are four metrics in this work, $\left(\right.$ Metric $\left._{1}\right)$ file size, $\left(\right.$ Metric $\left._{2}\right)$ CPSNR, $\left(\right.$ Metric $\left._{3}\right)$ FSIM, and $\left(\right.$ Metric $\left._{4}\right)$ S-CIELAB. $B P_{\mathrm{AB}}$ is the restored images with $A^{\text {th }} B P$ to $B^{\text {th }} B P$, where plane number $B$ is more important than plane number $A$. We generated seven $B P s$. $\mathrm{BP}_{17}, \mathrm{BP}_{27}, \mathrm{BP}_{37}, \mathrm{BP}_{47}, \mathrm{BP}_{57}, \mathrm{BP}_{67}$, and $\mathrm{BP}_{77}$.

Tables 4-7 shows the CMSE, CPSNR, S-CIELAB, and FSIM results for 20 images with seven BPs conditions.

Table 4. CMSE Results for 41 To $60^{\text {th }}$ LC Images

\begin{tabular}{|c|c|r|r|r|r|r|r|}
\hline $\begin{array}{c}\text { Image } \\
\text { number }\end{array}$ & $\mathrm{BP}_{77}$ & $\mathrm{BP}_{67}$ & \multicolumn{1}{c|}{$\mathrm{BP}_{57}$} & \multicolumn{1}{c|}{$\mathrm{BP}_{47}$} & $\mathrm{BP}_{37}$ & $\mathrm{BP}_{27}$ & $\mathrm{BP}_{17}$ \\
\hline 41 & 39752.23 & 6537.08 & 624.12 & 21.52 & 1.20 & 0.36 & 1.50 \\
\hline 42 & 36273.57 & 15689.62 & 4819.63 & 1356.74 & 164.44 & 11.33 & 1.20 \\
\hline 43 & 34890.27 & 8300.98 & 2904.36 & 881.89 & 135.30 & 8.57 & 1.50 \\
\hline 44 & 52853.66 & 40976.72 & 23028.02 & 3825.57 & 770.33 & 214.91 & 60.13 \\
\hline 45 & 37397.23 & 15457.92 & 1248.59 & 118.64 & 8.03 & 2.10 & 0.42 \\
\hline 46 & 42728.88 & 23263.02 & 11708.30 & 4541.47 & 1219.04 & 146.39 & 11.63 \\
\hline 47 & 37622.88 & 13023.44 & 5166.01 & 1353.86 & 123.01 & 2.88 & 0.48 \\
\hline 48 & 43836.60 & 21542.52 & 9918.38 & 2752.21 & 585.87 & 52.09 & 4.92 \\
\hline 49 & 38794.44 & 14155.08 & 5682.64 & 2554.98 & 453.21 & 21.58 & 1.74 \\
\hline 50 & 30739.12 & 10280.64 & 3063.88 & 566.15 & 49.58 & 3.78 & 0.54 \\
\hline 51 & 51314.80 & 32803.25 & 12919.49 & 3046.31 & 637.67 & 186.38 & 54.31 \\
\hline 52 & 29492.87 & 19299.15 & 6379.12 & 344.34 & 0.66 & 98.67 & 28.36 \\
\hline 53 & 38968.17 & 21429.75 & 8747.54 & 1333.78 & 23.80 & 0.78 & 0.18 \\
\hline 54 & 37962.72 & 13423.17 & 3148.70 & 244.59 & 59.29 & 7.43 & 1.20 \\
\hline 55 & 34581.00 & 17392.26 & 8251.89 & 2102.67 & 219.89 & 10.97 & 2.40 \\
\hline 56 & 28344.56 & 8685.73 & 2032.59 & 677.83 & 88.84 & 2.40 & 0.48 \\
\hline 57 & 44072.79 & 14500.50 & 3968.13 & 690.18 & 6.35 & 0.60 & 0.24 \\
\hline 58 & 36518.22 & 20688.50 & 11073.70 & 2369.20 & 210.48 & 9.41 & 1.08 \\
\hline 59 & 41860.24 & 15238.28 & 3289.10 & 271.74 & 17.09 & 2.58 & 0.42 \\
\hline 60 & 32577.96 & 8341.21 & 827.70 & 16.49 & 0.54 & 0.06 & 0.06 \\
\hline Avg. & 38529.11 & 17051.44 & 6440.09 & 1453.51 & 238.73 & 39.16 & 8.64 \\
\hline & & & & & & & \\
\hline
\end{tabular}




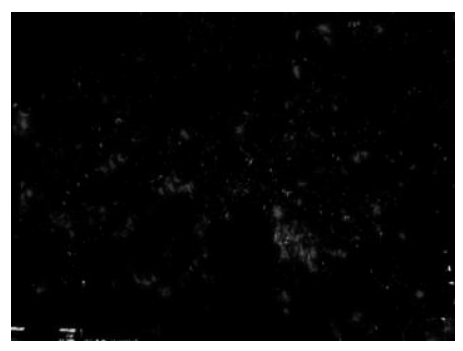

(a)

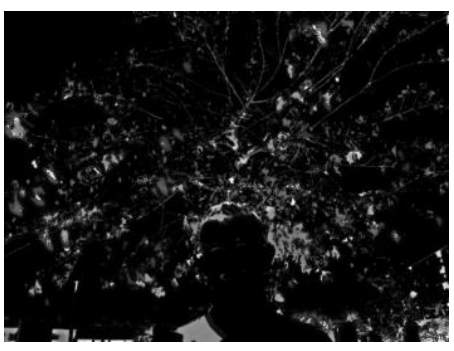

(b)

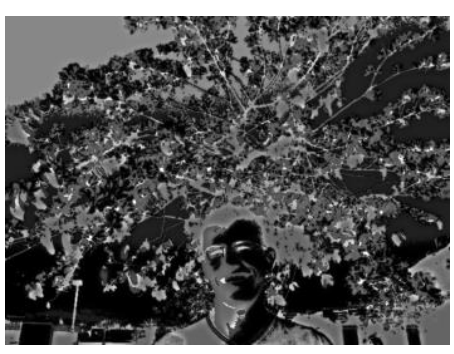

(c)

Figure 5. S-CIELAB Maps for the $(A) 2^{\text {nd }},(B) 1^{\text {st }}$, and (C) $0^{\text {th }}$ Bit Planes

Table 5. CPSNR Results for 41 to $60^{\text {th }}$ LC Images

\begin{tabular}{|c|r|r|r|r|r|r|r|}
\hline $\begin{array}{c}\text { Image } \\
\text { number }\end{array}$ & $\mathrm{BP}_{77}$ & $\mathrm{BP}_{67}$ & \multicolumn{1}{|c|}{$\mathrm{BP}_{57}$} & $\mathrm{BP}_{47}$ & $\mathrm{BP}_{37}$ & $\mathrm{BP}_{27}$ & $\mathrm{BP}_{17}$ \\
\hline 41 & 2.137 & 9.977 & 20.178 & 34.802 & 47.343 & 52.572 & 46.374 \\
\hline 42 & 2.535 & 6.175 & 11.301 & 16.806 & 25.971 & 37.588 & 47.343 \\
\hline 43 & 2.704 & 8.940 & 13.500 & 18.677 & 26.818 & 38.800 & 46.374 \\
\hline 44 & 0.900 & 2.005 & 4.508 & 12.304 & 19.264 & 24.808 & 30.340 \\
\hline 45 & 2.402 & 6.239 & 17.167 & 27.389 & 39.082 & 44.912 & 51.902 \\
\hline 46 & 1.824 & 4.464 & 7.446 & 11.559 & 17.271 & 26.476 & 37.475 \\
\hline 47 & 2.376 & 6.984 & 10.999 & 16.815 & 27.231 & 43.541 & 51.322 \\
\hline 48 & 1.712 & 4.798 & 8.166 & 13.734 & 20.453 & 30.963 & 41.215 \\
\hline 49 & 2.243 & 6.622 & 10.585 & 14.057 & 21.568 & 34.790 & 45.729 \\
\hline 50 & 3.254 & 8.011 & 13.268 & 20.602 & 31.178 & 42.360 & 50.811 \\
\hline 51 & 1.028 & 2.972 & 7.018 & 13.293 & 20.085 & 25.427 & 30.782 \\
\hline 52 & 3.434 & 5.275 & 10.083 & 22.761 & 49.939 & 31.578 & 37.557 \\
\hline 53 & 2.224 & 4.821 & 8.712 & 16.880 & 34.365 & 49.214 & 55.582 \\
\hline 54 & 2.337 & 6.852 & 13.149 & 24.246 & 30.401 & 39.419 & 47.343 \\
\hline 55 & 2.742 & 5.727 & 8.965 & 14.903 & 24.709 & 37.729 & 44.332 \\
\hline 56 & 3.606 & 8.743 & 15.050 & 19.820 & 28.645 & 44.332 & 51.322 \\
\hline 57 & 1.689 & 6.517 & 12.145 & 19.741 & 40.100 & 50.353 & 54.332 \\
\hline 58 & 2.506 & 4.974 & 7.688 & 14.385 & 24.899 & 38.394 & 47.800 \\
\hline 59 & 1.913 & 6.301 & 12.960 & 23.789 & 35.805 & 44.018 & 51.902 \\
\hline 60 & 3.002 & 8.919 & 18.952 & 35.960 & 50.811 & 60.353 & 60.353 \\
\hline Avg. & 2.328 & 6.266 & 11.592 & 19.626 & 30.797 & 39.881 & 46.510 \\
\hline
\end{tabular}

Figure 5 shows three S-CIELAB maps for the $2^{\text {nd }}, 1^{\text {st }}$, and $0^{\text {th }}$ bit planes of the test image. Figure 6 shows FSIM maps for all bit planes. 
Table 6. S-CIELAB Results for 41 to $60^{\text {th }}$ LC Images

\begin{tabular}{|c|c|c|c|c|c|c|c|}
\hline $\begin{array}{c}\text { Image } \\
\text { number }\end{array}$ & $\mathrm{BP}_{77}$ & $\mathrm{BP}_{67}$ & $\mathrm{BP}_{57}$ & $\mathrm{BP}_{47}$ & $\mathrm{BP}_{37}$ & $\mathrm{BP}_{27}$ & $\mathrm{BP}_{17}$ \\
\hline 41 & 0.3166 & 0.4777 & 0.8169 & 0.9508 & 0.9862 & 0.9932 & 1.0000 \\
\hline 42 & 0.5183 & 0.5965 & 0.7423 & 0.8664 & 0.9396 & 0.9669 & 0.9893 \\
\hline 43 & 0.3826 & 0.5332 & 0.8125 & 0.9024 & 0.9493 & 0.9796 & 0.9964 \\
\hline 44 & 0.6756 & 0.4994 & 0.5006 & 0.6800 & 0.9087 & 0.9658 & 0.9871 \\
\hline 45 & 0.4913 & 0.4104 & 0.7309 & 0.8710 & 0.9383 & 0.9759 & 0.9906 \\
\hline 46 & 0.5109 & 0.5326 & 0.5673 & 0.7727 & 0.8617 & 0.9177 & 0.9734 \\
\hline 47 & 0.4395 & 0.5975 & 0.7770 & 0.8406 & 0.8662 & 0.9647 & 0.9902 \\
\hline 48 & 0.4904 & 0.5413 & 0.6628 & 0.7813 & 0.8970 & 0.9588 & 0.9913 \\
\hline 49 & 0.3756 & 0.4383 & 0.6893 & 0.7918 & 0.8113 & 0.8411 & 0.9389 \\
\hline 50 & 0.3972 & 0.5037 & 0.7009 & 0.9044 & 0.9461 & 0.9859 & 0.9979 \\
\hline 51 & 0.5183 & 0.4524 & 0.5252 & 0.7239 & 0.8695 & 0.9684 & 0.9875 \\
\hline 52 & 0.6098 & 0.5399 & 0.6648 & 0.8299 & 0.9433 & 1.0000 & 1.0000 \\
\hline 53 & 0.3542 & 0.3362 & 0.4259 & 0.7448 & 0.8730 & 0.9052 & 0.9451 \\
\hline 54 & 0.3253 & 0.4373 & 0.6321 & 0.8798 & 0.9434 & 0.9750 & 0.9942 \\
\hline 55 & 0.4101 & 0.4949 & 0.6007 & 0.8079 & 0.9284 & 0.9689 & 0.9780 \\
\hline 56 & 0.5381 & 0.6161 & 0.8565 & 0.8974 & 0.9242 & 0.9823 & 0.9943 \\
\hline 57 & 0.3931 & 0.4347 & 0.7395 & 0.8474 & 0.8938 & 0.9788 & 0.9947 \\
\hline 58 & 0.5300 & 0.5068 & 0.5493 & 0.7457 & 0.8931 & 0.9299 & 0.9763 \\
\hline 59 & 0.3797 & 0.4160 & 0.6628 & 0.9086 & 0.9707 & 0.9904 & 0.9978 \\
\hline 60 & 0.5300 & 0.5068 & 0.5493 & 0.7457 & 0.8931 & 0.9299 & 0.9763 \\
\hline Avg. & 0.4593 & 0.4936 & 0.6603 & 0.8246 & 0.9118 & 0.9589 & 0.9850 \\
\hline
\end{tabular}

Table 7. Fsim Results for 41 To $60^{\text {th }}$ Lc Images

\begin{tabular}{|c|r|r|r|r|r|r|r|}
\hline $\begin{array}{c}\text { Image } \\
\text { number }\end{array}$ & \multicolumn{1}{|c|}{$\mathrm{BP}_{77}$} & \multicolumn{1}{|c|}{$\mathrm{BP}_{67}$} & \multicolumn{1}{c|}{$\mathrm{BP}_{57}$} & \multicolumn{1}{c|}{$\mathrm{BP}_{47}$} & $\mathrm{BP}_{37}$ & \multicolumn{1}{c|}{$\mathrm{BP}_{27}$} & $\mathrm{BP}_{17}$ \\
\hline 41 & 70.505 & 11.671 & 0.987 & 0.034 & 0.003 & 0.001 & 0.000 \\
\hline 42 & 58.768 & 28.010 & 9.214 & 2.404 & 0.228 & 0.015 & 0.003 \\
\hline 43 & 70.963 & 16.587 & 6.288 & 1.711 & 0.221 & 0.014 & 0.003 \\
\hline 44 & 84.683 & 72.044 & 35.941 & 5.579 & 1.163 & 0.332 & 0.100 \\
\hline 45 & 92.043 & 29.826 & 2.412 & 0.288 & 0.022 & 0.006 & 0.001 \\
\hline 46 & 71.830 & 40.861 & 19.267 & 7.211 & 1.913 & 0.185 & 0.016 \\
\hline 47 & 71.299 & 22.820 & 9.533 & 2.486 & 0.178 & 0.004 & 0.001 \\
\hline 48 & 95.087 & 35.529 & 16.899 & 4.811 & 0.922 & 0.065 & 0.009 \\
\hline 49 & 77.114 & 25.854 & 10.106 & 4.664 & 0.768 & 0.024 & 0.003 \\
\hline 50 & 51.404 & 15.640 & 4.698 & 0.895 & 0.059 & 0.006 & 0.001 \\
\hline 51 & 98.194 & 86.814 & 42.889 & 9.590 & 1.918 & 0.555 & 0.157 \\
\hline 52 & 47.305 & 32.308 & 10.656 & 0.453 & 0.001 & 0.000 & 0.000 \\
\hline 53 & 66.628 & 37.226 & 16.807 & 2.366 & 0.037 & 0.001 & 0.000 \\
\hline 54 & 66.962 & 24.630 & 5.201 & 0.420 & 0.092 & 0.013 & 0.002 \\
\hline 55 & 57.464 & 29.345 & 13.852 & 3.310 & 0.324 & 0.016 & 0.005 \\
\hline 56 & 47.775 & 15.106 & 3.740 & 1.248 & 0.121 & 0.004 & 0.001 \\
\hline 57 & 76.928 & 24.671 & 6.857 & 1.060 & 0.008 & 0.001 & 0.000 \\
\hline 58 & 62.936 & 44.364 & 21.413 & 3.969 & 0.303 & 0.014 & 0.002 \\
\hline 59 & 71.585 & 31.865 & 5.817 & 0.431 & 0.037 & 0.007 & 0.001 \\
\hline 60 & 67.838 & 16.607 & 2.060 & 0.035 & 0.002 & 0.000 & 0.000 \\
\hline Avg. & 70.366 & 32.089 & 12.232 & 2.648 & 0.416 & 0.063 & 0.015 \\
\hline
\end{tabular}




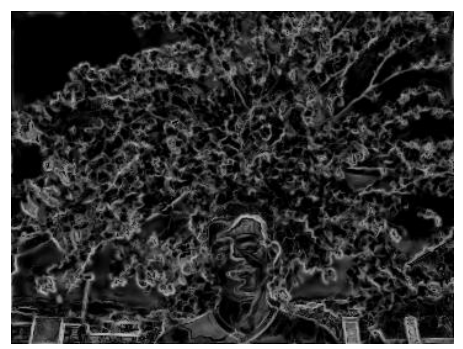

(a)

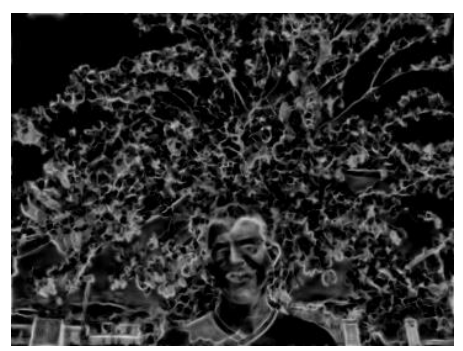

(d)

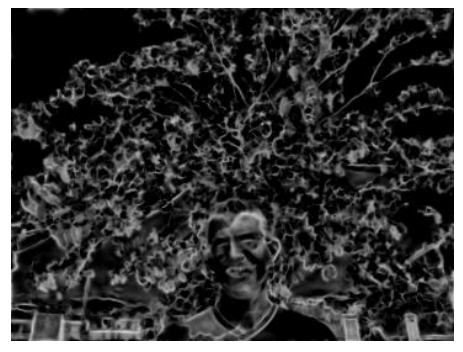

(g)

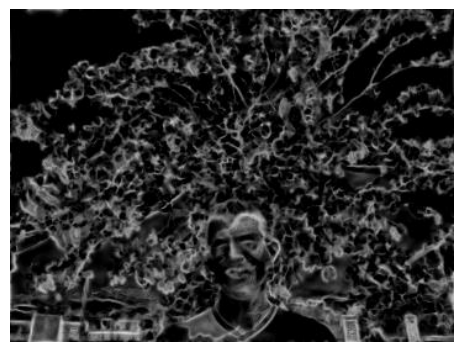

(b)

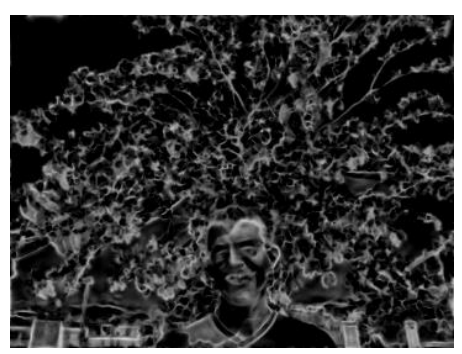

(e)

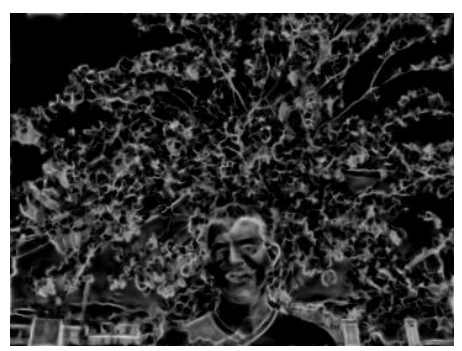

(h)

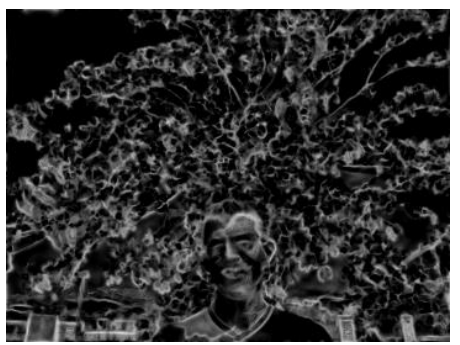

(c)

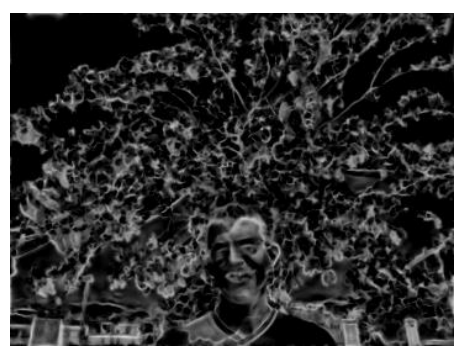

(f)

Figure 6. FSIM Maps with $A^{\text {th }}$ BP To $B^{\text {th }} B P$ on $80^{\text {th }}$ LC Image: (A) $B P_{77}$, (B) $\mathrm{BP}_{67},(\mathrm{C}) \mathrm{BP}_{57}$, (D) $\mathrm{BP}_{47},(\mathrm{E}) \mathrm{BP}_{37},(\mathrm{~F}) \mathrm{BP}_{27},(\mathrm{G}) \mathrm{BP}_{17}$, And (H) $\mathrm{BP}_{07 .}$

Figure 7 shows independent bit plane image while Figure 8 shows visual comparison of $\mathrm{BP}_{07}$ to $\mathrm{BP}_{77}$ result images.

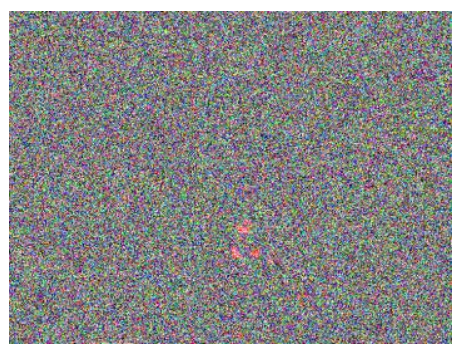

(a)

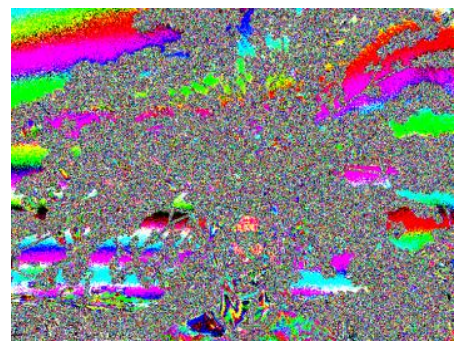

(d)

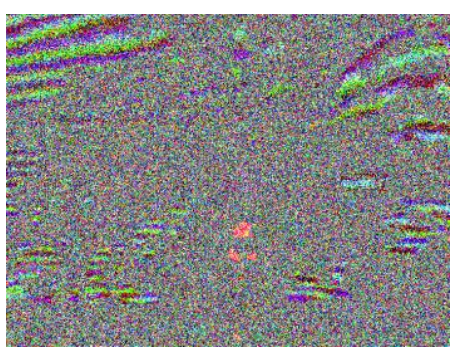

(b)

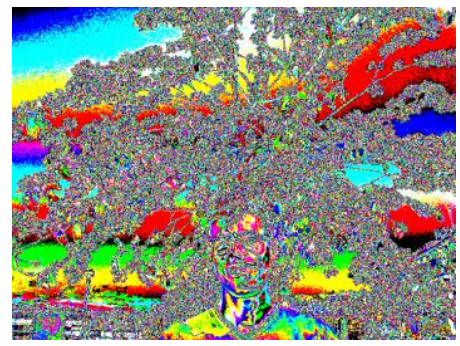

(e)

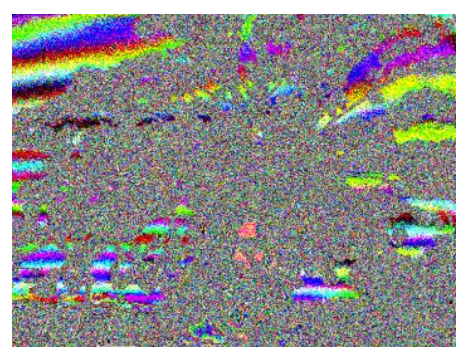

(c)

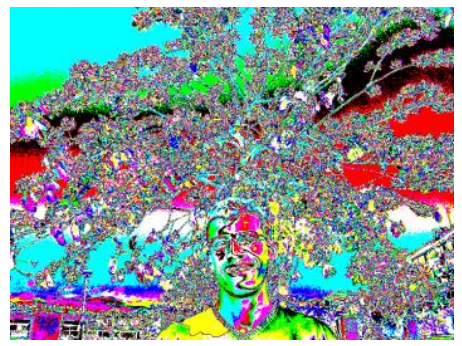

(f) 


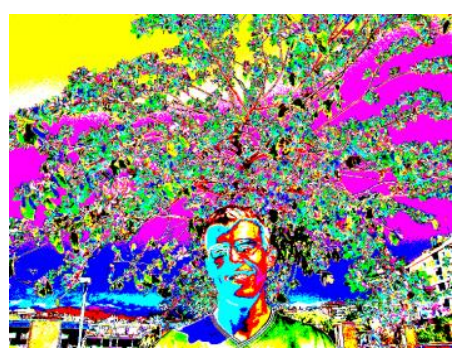

(g)

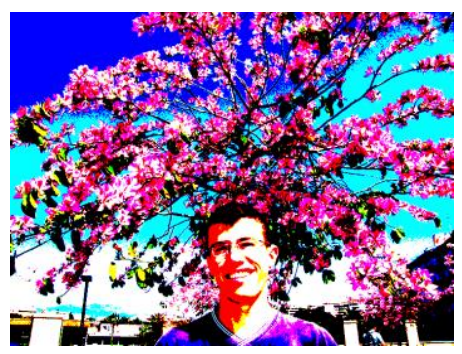

(h)

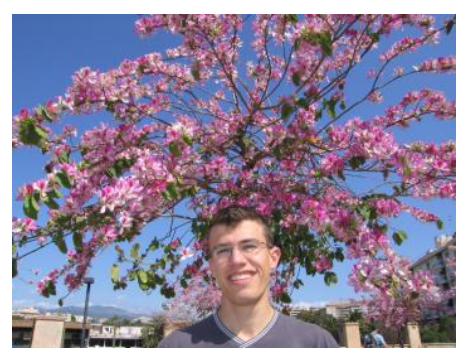

(i)

Figure 7. Bit Plane Image: (A) $0^{\text {th }}$ Bit Plane, (B) $1^{\text {st }}$ Bit Plane, (C) $2^{\text {nd }}$ Bit Plane, (D) $3^{\text {rd }}$ Bit Plane, (E) $4^{\text {th }}$ Bit Plane, (F) $5^{\text {th }}$ Bit Plane, (G) $6^{\text {th }}$ Bit Plane, (H) $7^{\text {th }}$ Bit Plane, And (I) Original Image.

\section{Conclusions}

When signals are described in digital format, signal can be decomposed in sliced bits. This paper analyzed the tradeoff between image size and performance. The performance was measured by four metrics CMSE, CPSNR, S-CIELAB, and FSIM. Experimental results show the performance in each metrics.

\section{Acknowledgements}

This work was supported by the National Research Foundation of Korea(NRF) Grant funded by the Korean Government(MSIP)(2014025627).

This paper is a revised and expanded version of a paper entitled "Performance Aware Bit Plane Assignment" presented at CIA2016.

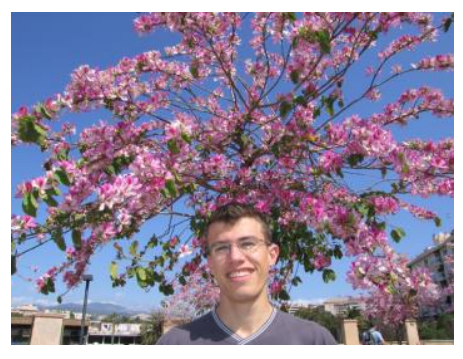

(a)

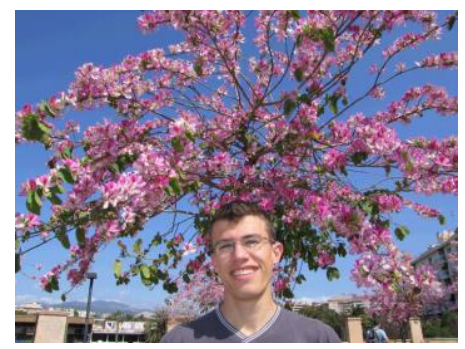

(d)

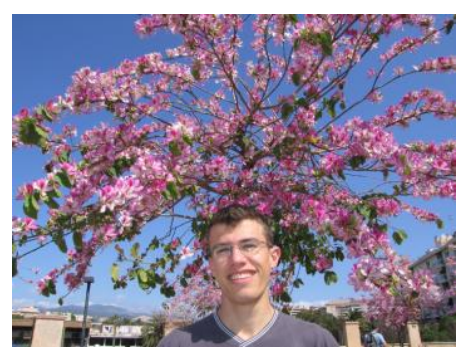

(b)

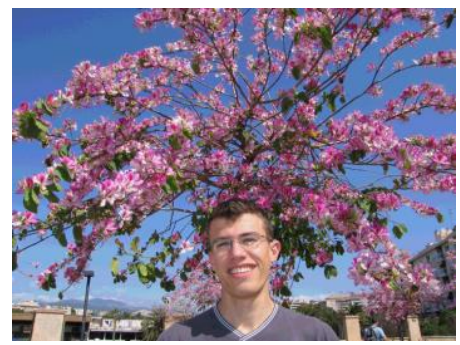

(e)

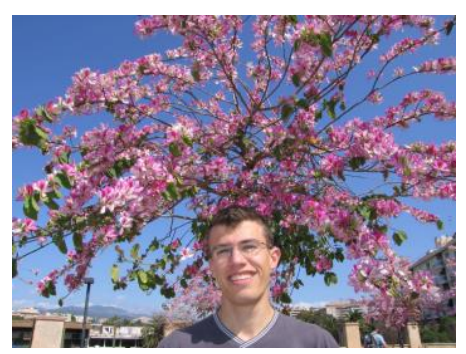

(c)

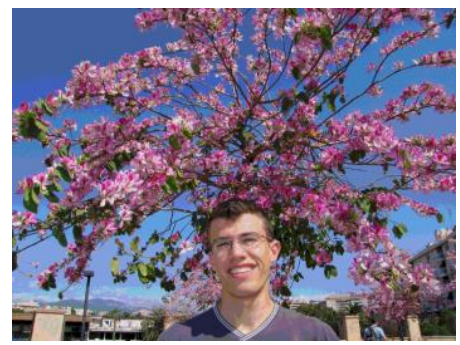

(f) 


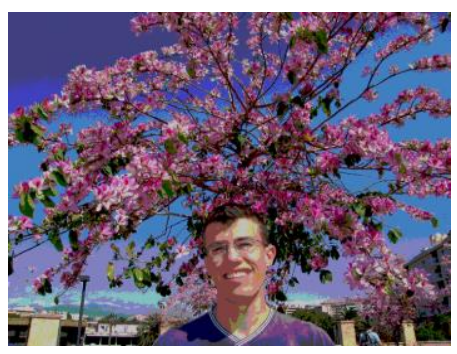

(g)

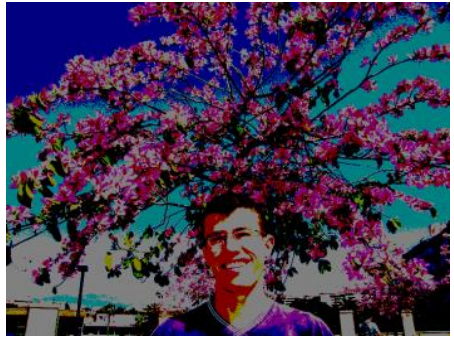

(h)

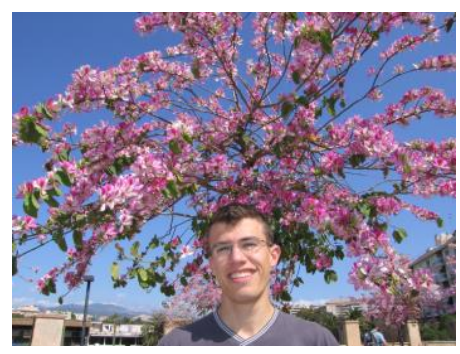

(i)

Figure 8. Restored Images with $A^{\text {th }} B P$ To $B^{\text {th }} B P$ on $80^{\text {th }} L C$ Image: $(A) B P_{77}$, (B) $\mathrm{BP}_{67}$, (C) $\mathrm{BP}_{57}$, (D) $\mathrm{BP}_{47}$, (E) $\mathrm{BP}_{37}$, (F) $\mathrm{BP}_{27}$, (G) $\mathrm{BP}_{17}$, (H) $\mathrm{BP}_{07}$, And (I) Original Image

\section{References}

[1] G.C. Langelaar, I. Setyawan and R. L. Lagendijk, "Watermarking digital image and video data", IEEE Signal Processing Magazine, (2000), pp.20-46.

[2] S. Jun-Lee, Y.-H. Lee, H. Ahn and S. B. Rhee, "Color image descriptor using wavelet correlogram", The 23rd international conference on Circuits/systems, computers and communication, (2008).

[3] M. H. Sedaaghi and S. Yousefi, "Morphological watermarking", IEE Electronics Letters, vol.41 no.10, (2005), pp.591-593.

[4] R.C. Gonzalez and R.E. Woods, Digital Image Processing 2nd ed.,Prentice Hall, Inc., New Jersey, (2002).

[5] G. Qiu, “Colour Image Indexing Using BTC”, IEEE Transition on Image Processing, vol. no 12, (2003).

[6] M. Petrou and C. Petrou, "Image Processing: The Fundamentals", Wiley, Amsterdam, (2010).

[7] P. Suresh,R.M.D. Sundaram and A. Arumugam, "Feature Extraction in Compressed Domain for Content Based Image Retrieval, "International Conference on Advanced Computer Theory and Engineering, (2008).

[8] C. H. Yeh and C. J. Kuo, "Digital watermarking through quasi marrays", Proc.of 25th annual IEE Conf. on Industrial Electronics Society, vol.1, (1999), pp.459-461.

[9] N.S.T. Sai, "Ravindra patil,"Image Retrival usng Equalized Histogram Image Bins Moment", International Joint Journal Conference in Engneering, ,IJUCE, Trivandrum,India, (2010).

[10] A. W. M. Smeulders, M. Worring, S. Santini, A. Gupta and R. Jain, "Content-based image retrieval at the end of the early years", IEEE Transaction on Pattern Analysis and Machine Intelligence (PAMI), vol. 22, no. 12, (2000), pp. 1349-1380.

[11] R.G. van Schyndel, A.Z. Tirkel and C.F. Osborne, "A digital watermark", Proc. IEEE Int. Conf. on Image Processing, vol.2, (1994), pp. 86-90.

[12] W.Y. Ma and B.S. Manjunath, "Pictorial Queries: Combining Feature Extraction with Database Search", Technical Report 18, Dept. of Electrical Eng., Univ. of California at Santa Barbara, (1994).

[13] W.K. Pratt, "Digital image processing", A Wiley Interscience Publication, (1991).

[14] A. Gupta and R. Jain, " "Visual Information Retrieval", Comm. ACM, vol. 40, no. 5, (1997).

[15] K.C. Ting, D.B.L. Bong and Y.C. Wang, "Performance Analysis of Single and Combined Bit-Planes Feature Extraction for Recognition in Face Expression Database", Proceedings of the International Conference on Computer and Communication Engineering 2008,May 13-15, 2008 Kuala Lumpur, Malaysia.

[16] C.E. Jacobs, A. Finkelstein and D.H. Salesin, "Fast Multiresolution Image Querying", Proc. SIGGRAPH 95, (995).

[17] W.J.Z. Wang, G. Wiederhold, O. Firschei and S.X. Wei, "Wavelet Based Image Indexing Techniques with Partial Sketch Retrieval Capability", J. Digital Libraries, (1997).

[18] M. Mohammed Sathik, "Feature Extracton on ColorED x-Ray Images by Bit-plane Slicing Technique", International Journal of Engineering Science and Technology, vol. 2, no.7, (2010), pp. 2820-2824.

[19] G. Haldankar, A. Tikare and J. Patil, "Converting Gray Scale Image to Color Image", in Proceedings of SPIT-IEEE Colloquium and International Conference, Mumbai, India, Vol. 1, no. 189.

[20] N. Ravia S. Parveen and M. Mohamed Sathik, "Feature Extraction by Bit Plane Slicing Technique", in International Journal of Computing, Communication and Information System, vol. 1.

[21] K. Hirata and T. Kato, "Qauery by Visual Example”, Advances in Database Technology EDBT '92, Third Int'l Conf. Extending Database Technology, (1992).

[22] W.Y. Ma and B.S. Manjunath, "Pictorial Queries: Combining Feature Extraction with Database Search", Technical Report 18, Dept. of Electrical Eng., Univ. of California at Santa Barbara, (1994). 
[23] A Gupata and R. Jain, "Visual Information Retrieval", Comman. ACM, vol.40, no.5, (1997), pp. 70- 79.

[24] X. Zhang, D. Silverstein, J. Farrell and B. Wandell, "Color image quality metric S-CIELAB and its application to halftone texture visibility", IEEE Compcon, vol. 97, (1997).

[25] L. Zhang, L. Zhang, X. Mou and D. Zhang, "FSIM: A Feature Similarity Index for Image Quality Assessment”, IEEE Trans. Image Processing, vol. 20, no. 8, (2011), pp. 2378-2386.

[26] G. Jeon, "Contrast Intensification in NTSC YIQ," IJCA vol. 6, no. 4, (2013), pp. 157-166.

[27] G. Jeon, "Measuring and Comparison of Edge Detectors in Color Spaces", IJCA vol. 6, no. 5, (2013), pp. 21-30.

\section{Author}

Gwanggil Jeon, he received the BS, MS, and $\mathrm{PhD}$ (summa cum laude) degrees in Department of Electronics and Computer Engineering from Hanyang University, Seoul, Korea, in 2003, 2005, and 2008, respectively.

From 2008 to 2009, he was with the Department of Electronics and Computer Engineering, Hanyang University, from 2009 to 2011, he was with the School of Information Technology and Engineering (SITE), University of Ottawa, as a postdoctoral fellow, and from 2011 to 2012, he was with the Graduate School of Science \& Technology, Niigata University, as an assistant professor. He is currently an associate professor with the Department of Embedded Systems Engineering, Incheon National University, Incheon, Korea. His research interests fall under the umbrella of image processing, particularly image compression, motion estimation, demosaicking, and image enhancement as well as computational intelligence such as fuzzy and rough sets theories.

He was the recipient of the IEEE Chester Sall Award in 2007 and the 2008 ETRI Journal Paper Award. 